\title{
Article \\ Structural Diversities of a Series of Cd(II) Coordination Complexes Based on a Flexible Tripodal N-donor Ligand
}

\author{
Huarui Wang *(i) and Jianhua Qin
}

check for

updates

Citation: Wang, H.; Qin, J. Structural

Diversities of a Series of Cd(II)

Coordination Complexes Based on a

Flexible Tripodal N-donor Ligand.

Crystals 2022, 12, 53. https://

doi.org/10.3390/cryst12010053

Academic Editors: Ana M.

Garcia-Deibe, Antonio

Rodriguez-Dieguez and Jesús

Sanmartín-Matalobos

Received: 15 November 2021

Accepted: 23 December 2021

Published: 31 December 2021

Publisher's Note: MDPI stays neutral with regard to jurisdictional claims in published maps and institutional affiliations.

Copyright: (C) 2021 by the authors. Licensee MDPI, Basel, Switzerland. This article is an open access article distributed under the terms and conditions of the Creative Commons Attribution (CC BY) license (https:// creativecommons.org/licenses/by/ $4.0 /)$.
Henan Key Laboratory of Function-Oriented Porous Materials, College of Chemistry and Chemical Engineering, Luoyang Normal University, Luoyang 471934,China; jh_q128105@126.com

* Correspondence: whr@lynu.edu.cn; Tel.: +86-379-6861-8321

\begin{abstract}
Three $\mathrm{Cd}(\mathrm{II})$ coordination complexes with unique structures and topologies, namely, $\left\{[\mathrm{Cd}(\mathrm{tttmb})(\mathrm{Hbtc})] \cdot 5 \mathrm{H}_{2} \mathrm{O}\right\}_{\mathrm{n}} \quad(\mathbf{1}), \quad\left\{\left[\mathrm{Cd}(\mathrm{tttmb})(\mathrm{m} \text {-phda })\left(\mathrm{H}_{2} \mathrm{O}\right)\right] \cdot 2 \mathrm{H}_{2} \mathrm{O}\right\}_{\mathrm{n}} \quad$ (2), $\quad$ and $\left\{[\mathrm{Cd}(\mathrm{tttmb})(\mathrm{o}-\mathrm{cpla})] \cdot\left(\mathrm{CH}_{3} \mathrm{CN}\right) \cdot\left(\mathrm{H}_{2} \mathrm{O}\right)_{1.5}\right\}_{\mathrm{n}}$ (3), have been successfully synthesized under hydro(solvo)thermally condition based on a flexible tripodal $\mathrm{N}$-contained ligand 1,3,5-tris $(1,2,4$ triazol-1-ylmethyl)-2,4,6-trimethylbenzene (tttmb) and aromatic polycarboxylate acids $\left(\mathrm{H}_{3} \mathrm{btc}=1,2,4\right.$ benzenetricarboxylic acid, $\mathrm{m}-\mathrm{H}_{2}$ phda $=1,3$-phenylenediacetic acid and o- $\mathrm{H}_{2} \mathrm{cpla}=$ Homophthalic acid). Complexes 1-3 were characterized by elemental analysis, IR spectroscopy, X-ray single-crystal diffraction and thermogravimetric analyses. 1 crystallize in the orthorhombic chiral space group $P 2{ }_{1} 2_{1} 2_{1}$ and feature 3D coordination networks. 2 reveals a $2 \mathrm{D}$ ladder-like structure with $(4,4)$ topology containing alternating $\mathrm{Cd}(\mathrm{II}) / \mathrm{m}$-phda ${ }^{2-}$ left- and right-handed helical motifs. 3 exhibits a 3D net with $\left(6^{3}\right)\left(6^{6}\right)\left(7 \cdot 8^{2}\right)$ topology. The structural and dimensional diversity of these complexes not only indicates that the flexible ligand ttmb exhibits strong coordination ability and diverse coordination modes, but also shows that aromatic polycarboxylates play important roles in constructing the frameworks of complexes. Moreover, the different photoluminescence behaviors of 1-3 have been studied in the solid state.
\end{abstract}

Keywords: coordination polymer; chiral space group; ladder-like structure; luminescence

\section{Introduction}

In order to obtain unique MOFs with interesting properties, many flexible ligands have been widely employed as linkers of metal ions, specifically flexible N-donor bridging ligands [1-5]. The introduction of N-rich functional ligands into MOFs is an available strategy to realize the functionalization of materials, as the accessible $\mathrm{N}$ sites, such as $\mathrm{NH}_{2}$ groups, imidazole, and N-rich triazole units, can enhance their catalytic performance and gas adsorption capacity [6-10]. Triazole containing ligands usually have strong coordination ability to transition metal ion centers For example, as multifunctional organic linkers, 1,2,4-triazole and its derivatives can possess a combination of the advantages of the coordination geometries of both pyrazole and imidazole, and provide more potential coordination sites [11,12]. Compared with the rigid ligands, conformation of the flexible 1,2,4-triazole-containing ligands can often be influenced by exterior factors; thus, specific structures and functions can be used to coordinate different metal ions. At the same time, the flexible ligand itself is easy to rotate and twist in the process of self-assembly.

Meanwhile, polycarboxylate organic ligands, which have good coordination ability and various coordination modes, have proven to be excellent structural contributors [13-15]. The coordination complexes based on the mixed 1,2,4-triazole-containing and polycarboxylate-based ligands were explored with complicated coordination environments. A series of 1,2,4-triazole-containing ligands with distinct orientation and flexibility were reported, as 1,4-bis(1,2,4-triazol-1-ylmethyl)-benzene (bbtz), 4,4'-bis(1,2,4-triazol-1ylmethyl)biphenyl (btmb), 1,4-bis(1,2,4-triazol-1-yl)butane (btb), 1,3,5-tris(1,2,4-triazol-1- 
ylmethyl)-2,4,6-trimethylbenzene (tttmb) [16-26]. For further understanding of the coordination principle, systematic study was carried out for 1,2,4-triazole-containing tripodal ligand $\mathrm{Cd}(\mathrm{II})$ coordination complexes, and three $\mathrm{Cd}(\mathrm{II})$ coordination complexes, namely, $\left\{[\mathrm{Cd}(\mathrm{tttmb})(\mathrm{Hbtc})] \cdot 5 \mathrm{H}_{2} \mathrm{O}\right\}_{n}\left(\mathbf{1}, \mathrm{H}_{3} \mathrm{btc}=1,2,4\right.$-benzenetricarboxylic acid $),\{[\mathrm{Cd}(\mathrm{tttmb})(m-$ phda) $\left.\left.\left(\mathrm{H}_{2} \mathrm{O}\right)\right] \cdot 2 \mathrm{H}_{2} \mathrm{O}\right\}_{n}\left(2, \mathrm{~m}-\mathrm{H}_{2}\right.$ phda $=1,3$-phenylenediacetic acid $)$, and $\{[\mathrm{Cd}(\mathrm{tttmb})(o-$ cpla) $\left.] \cdot\left(\mathrm{CH}_{3} \mathrm{CN}\right) \cdot\left(\mathrm{H}_{2} \mathrm{O}\right)_{1.5}\right\}_{n}\left(3, \mathrm{o}-\mathrm{H}_{2} \mathrm{cpla}=\right.$ homophthalic acid $)$ were successfully synthesized. In addition, the crystal structures and topology of 1-3 were characterized and discussed. Moreover, the thermal stability and photoluminescence properties of $\mathbf{1}-\mathbf{3}$ were also studied.

\section{Materials and Methods}

\subsection{Reagents and Instruments}

All reagents and solvents, except for tttmb, were purchased from commercial sources and used directly without further purifications. The ligand $\mathrm{tttmb}$ was synthesized according to the literature (see the Supplementary Information for a more detailed description of the synthesis) [21]. Infrared spectra $\left(4000-400 \mathrm{~cm}^{-1}\right)$ were recorded on a Bruker Tensor 27 spectrometer (Bruker, Bremen, Germany) with KBr pellets. C, H, N elemental analyses were carried out on a Flash EA 1112 elemental analyzer (Nicolet, Ventura, CA, USA). Powder X-ray diffraction data were collected on a PANalytical X'Pert PRO diffractometer (PANalytical, Almelo, The Netherlands) with $\mathrm{Cu}-\mathrm{K} \alpha$ radiation $(\lambda=1.5406 \AA)$. Thermal analyses were performed on a Netzsch STA 449C thermal analyzer (Netzsch, Selb, Germany) from room temperature at a heating rate of $10{ }^{\circ} \mathrm{C} \mathrm{min}-1$ in air. The solid-state luminescent spectra were performed on a Hitachi 850 fluorescent spectrometer (Hitachi, Chiyoda, Japan) using Xe lamp as the light source.

\subsection{Single-Crystal X-ray Diffraction}

Determination of the crystal structures of 1-3 by X-ray diffractions was carried on a Rigaku Saturn 724 CCD diffractometer with MoK $\alpha$ radiation $(\lambda=0.71073 \AA)$ at $290 \pm 1 \mathrm{~K}$. The structures were solved with the Olex2 program as an interface, together with the SHELXT and SHELXL programs $[27,28]$. In the refinement, all non-hydrogen atoms were provided with anisotropic displacement parameters and all hydrogen atoms were treated by a riding model. CCDC 1901158, 1901159, and 1901160 contains the supplementary crystallo-graphic data for $\mathbf{1}, \mathbf{2}$, and $\mathbf{3}$. Crystallographic crystal data and structure processing parameters for 1-3 are summarized in Table 1 . Selected bond lengths and bond angles of 1-3 are listed in Table S1.

Table 1. Crystallographic parameters for compounds 1-3.

\begin{tabular}{cccc}
\hline Compound & $\mathbf{1}$ & $\mathbf{2}$ & $\mathbf{3}$ \\
\hline Formula & $\mathrm{C}_{27} \mathrm{H}_{35} \mathrm{~N}_{9} \mathrm{O}_{11} \mathrm{Cd}$ & $\mathrm{C}_{28} \mathrm{H}_{35} \mathrm{~N}_{9} \mathrm{O}_{7} \mathrm{Cd}$ & $\mathrm{C}_{29} \mathrm{H}_{30} \mathrm{~N}_{10} \mathrm{O}_{5} \mathrm{Cd}$ \\
Formula weight & 774.0319 & 722.05 & 711.03 \\
Crystal system & orthorhombic & monoclinic & monoclinic \\
Space group & $P 2{ }_{1}{ }_{1} 21$ & $\mathrm{C} 2 / \mathrm{c}$ & $\mathrm{C} 2 / \mathrm{c}$ \\
$a(\AA)$ & $11.281(2)$ & $30.639(6)$ & $19.825(4)$ \\
$b(\AA)$ & $13.642(3)$ & $12.889(3)$ & $13.620(3)$ \\
$c(\AA)$ & $22.337(5)$ & $19.841(4)$ & $23.123(5)$ \\
$\alpha\left(^{\circ}\right)$ & 90.00 & 90.00 & 90.00 \\
$\beta\left(^{\circ}\right)$ & 90.00 & $121.18(3)$ & $95.43(3)$ \\
$\gamma\left({ }^{\circ}\right)$ & 90.00 & 90.00 & 90.00 \\
$V\left(\AA^{3}\right)$ & $3437.7(12)$ & $6704(2)$ & $6216(2)$ \\
$Z$ & 4 & 8 & 8 \\
$D$ calc $\left(\mathrm{g} \cdot \mathrm{cm}^{-3}\right)$ & 1.476 & 1.431 & 1.520 \\
$\mu\left(\mathrm{mm}^{-1}\right)$ & 0.703 & 0.707 & 0.758 \\
\hline
\end{tabular}


Table 1. Cont.

\begin{tabular}{cccc}
\hline Compound & $\mathbf{1}$ & $\mathbf{2}$ & $\mathbf{3}$ \\
\hline$F(000)$ & 1544 & 2960 & 2944 \\
$R_{1} \mathrm{a}, w R_{2} \mathrm{~b}$ & $0.0758,0.1916$ & $0.0747,0.1686$ & $0.0630,0.1571$ \\
$(\mathrm{I}>2 \sigma(\mathrm{I}))$ & $0.0864,0.2002$ & $0.0912,0.1800$ & $0.0803,0.1806$ \\
$R_{1}, w R_{2}(\mathrm{all}$ data $)$ & 1.061 & 1.140 & 1.180 \\
GOF & ${ }^{\mathrm{a}} R=\left[\sum|| \mathrm{F}_{0}|-| \mathrm{F}_{\mathrm{C}}|| / \sum\left|\mathrm{F}_{0}\right|\right]^{\mathrm{b}} R_{W}=\sum_{W}\left[\left|\mathrm{~F}_{0}^{2}-\mathrm{F}_{\mathrm{C}}^{2}\right|^{2} / \sum_{W}\left(\left|\mathrm{~F}_{W}\right|^{2}\right)^{2}\right]^{1 / 2}$ &
\end{tabular}

${ }^{\mathrm{a}} R=\left[\sum|| \mathrm{F}_{0}|-| \mathrm{F}_{\mathrm{c}}|| / \sum\left|\mathrm{F}_{0}\right|\right],{ }^{\mathrm{b}} R_{W}=\sum_{\mathrm{W}}\left[\left|\mathrm{F}_{0}{ }^{2}-\mathrm{Fc}^{2}\right|^{2} / \sum \mathrm{W}\left(\left|\mathrm{F}_{\mathrm{w}}\right|^{2}\right)^{2}\right]^{1 / 2}$.

\subsection{Preparation of Compounds $\mathbf{1}-\mathbf{3}$}

2.3.1. Synthesis of $\left\{[\mathrm{Cd}(\mathrm{tttmb})(\mathrm{Hbtc})] \cdot 5 \mathrm{H}_{2} \mathrm{O}\right\}_{\mathrm{n}}(\mathbf{1})$

$\mathrm{Cd}\left(\mathrm{CH}_{3} \mathrm{COO}\right)_{2} \cdot 2 \mathrm{H}_{2} \mathrm{O}(0.023 \mathrm{~g}, 0.1 \mathrm{mmol}), \mathrm{tttmb}(0.037 \mathrm{~g}, 0.1 \mathrm{mmol}), \mathrm{H}_{3}$ btc $(0.021 \mathrm{~g}$, $0.1 \mathrm{mmol})$ were dissolved in $\mathrm{H}_{2} \mathrm{O}(8 \mathrm{~mL})$. Following this, the mixed solution was kept in a Teflon-lined autoclave at $403 \mathrm{~K}$ for 3 days. Then, the reaction mixture was cooled down to room temperature, and the colourless crystals were obtained with a yield of $75 \%$ (based on Cd). Anal. Calc. For $\mathrm{C}_{27} \mathrm{H}_{35} \mathrm{~N}_{9} \mathrm{O}_{11} \mathrm{Cd}(\%)$ : C, 41.9; $\mathrm{H}, 4.6$; N, 16.3. Found: $\mathrm{C}, 41.9 ; \mathrm{H}, 4.4$; $\mathrm{N}$, 16.6. IR (KBr, cm $\left.{ }^{-1}\right)$ : $3440(\mathrm{w}), 3407$ (w), 2958 (m), 2924 (s), 2360 (s), 2341 (s), 1748 (s), 1717 (s), 1647 (s), 1559 (s), 1436 (s), 1386 (s), 1134 (s), 669 (s), 515 (m), 449 (m).

\subsubsection{Synthesis of $\left\{\left[\mathrm{Cd}(\mathrm{tttmb})(\mathrm{m} \text {-phda })\left(\mathrm{H}_{2} \mathrm{O}\right)\right] \cdot 2 \mathrm{H}_{2} \mathrm{O}\right\}_{n}(2)$}

Compound 2 was obtained using a similar method as 1, except $\mathrm{m}-\mathrm{H}_{2}$ phda $(0.019 \mathrm{~g}$, $0.1 \mathrm{mmol}$ ) was used instead of $\mathrm{H}_{3}$ btc. Colorless crystals were collected with a yield of $60 \%$ based on Cd. Anal. Calc. For $\mathrm{C}_{28} \mathrm{H}_{35} \mathrm{~N}_{9} \mathrm{O}_{7} \mathrm{Cd}(\%)$ : C, 46.6; H, 4.9; N, 17.5. Found: C, 46.9; $\mathrm{H}, 4.4$; N, 16.9. IR (KBr, cm $\left.{ }^{-1}\right)$ : 3416 (w), 3125 (m), 2925 (m), 1561 (s), 1396 (s), 1261 (s), 1019 (s), 889 (m), 816 (m), 749 (s), 633 (s), 525 (m), 438 (w).

\subsubsection{Synthesis of $\left\{[\mathrm{Cd}(\mathrm{tttmb})(\mathrm{o}-\mathrm{cpla})] \cdot\left(\mathrm{CH}_{3} \mathrm{CN}\right) \cdot\left(\mathrm{H}_{2} \mathrm{O}\right)\right\}_{\mathrm{n}}(3)$}

$\mathrm{Cd}\left(\mathrm{CH}_{3} \mathrm{COO}\right)_{2} \cdot 2 \mathrm{H}_{2} \mathrm{O}(0.023 \mathrm{~g}, 0.1 \mathrm{mmol}), \mathrm{tttmb}(0.037 \mathrm{~g}, 0.1 \mathrm{mmol}), \mathrm{o}-\mathrm{H}_{2}$ cpla $(0.018 \mathrm{~g}$, $0.1 \mathrm{mmol})$ were dissolved in a mixture of $\mathrm{H}_{2} \mathrm{O}(10 \mathrm{~mL})$ and $\mathrm{CH}_{3} \mathrm{CN}(1 \mathrm{~mL})$. Following this, the mixed solution was kept in a $25 \mathrm{~mL}$ Teflon-lined autoclave and heated at $403 \mathrm{~K}$ for 3 days. After the mixture had been cooled to room temperature, colourless crystals were obtained with a yield of $75 \%$ based on Cd. Anal. Calc. $\mathrm{C}_{29} \mathrm{H}_{33} \mathrm{~N}_{10} \mathrm{O}_{5.5} \mathrm{Cd}$ (\%): C, 49.0; $\mathrm{H}$, 4.3; N, 19.7. Found: C, 47.3; H, 4.8; N, 18.5. IR (KBr, cm $\left.{ }^{-1}\right): 3424(\mathrm{w}), 3134(\mathrm{~m}), 3092(\mathrm{~m})$, 2923 (m), 1586 (s), 1519 (s), 1445 (m), 1389 (s), 1277 (s), 1134 (s), 1010 (s), 983 (s), 737 (s), $675(\mathrm{~s})$.

\section{Results and Discussion}

\subsection{Structural Description}

\subsubsection{Crystal Structure of $\mathbf{1}$}

1 is a $3 \mathrm{D}$ net and also crystallizes in the chiral orthorhombic space group $P 2{ }_{1} 2_{1} 2_{1}$ with Flack parameter of -0.01 (6) refined from the X-ray data. The asymmetric unit of 1 consists of one $\mathrm{Cd}(\mathrm{II})$ ion, one tttmb ligand, one $\mathrm{Hbtc}^{2-}$ ligand and five crystallization water molecule. As shown in Figure 1a, the Cd(II) ion is seven-coordinated, adopting a distorted pentagonal bipyramidal geometry via coordinating to four carboxylate oxygen atoms and three nitrogen atoms. The $\mathrm{Cd}-\mathrm{N}$ distances are in the range of $2.303-2.352 \AA$ and the Cd-O bond lengths vary from $2.410-2.460 \AA$. 


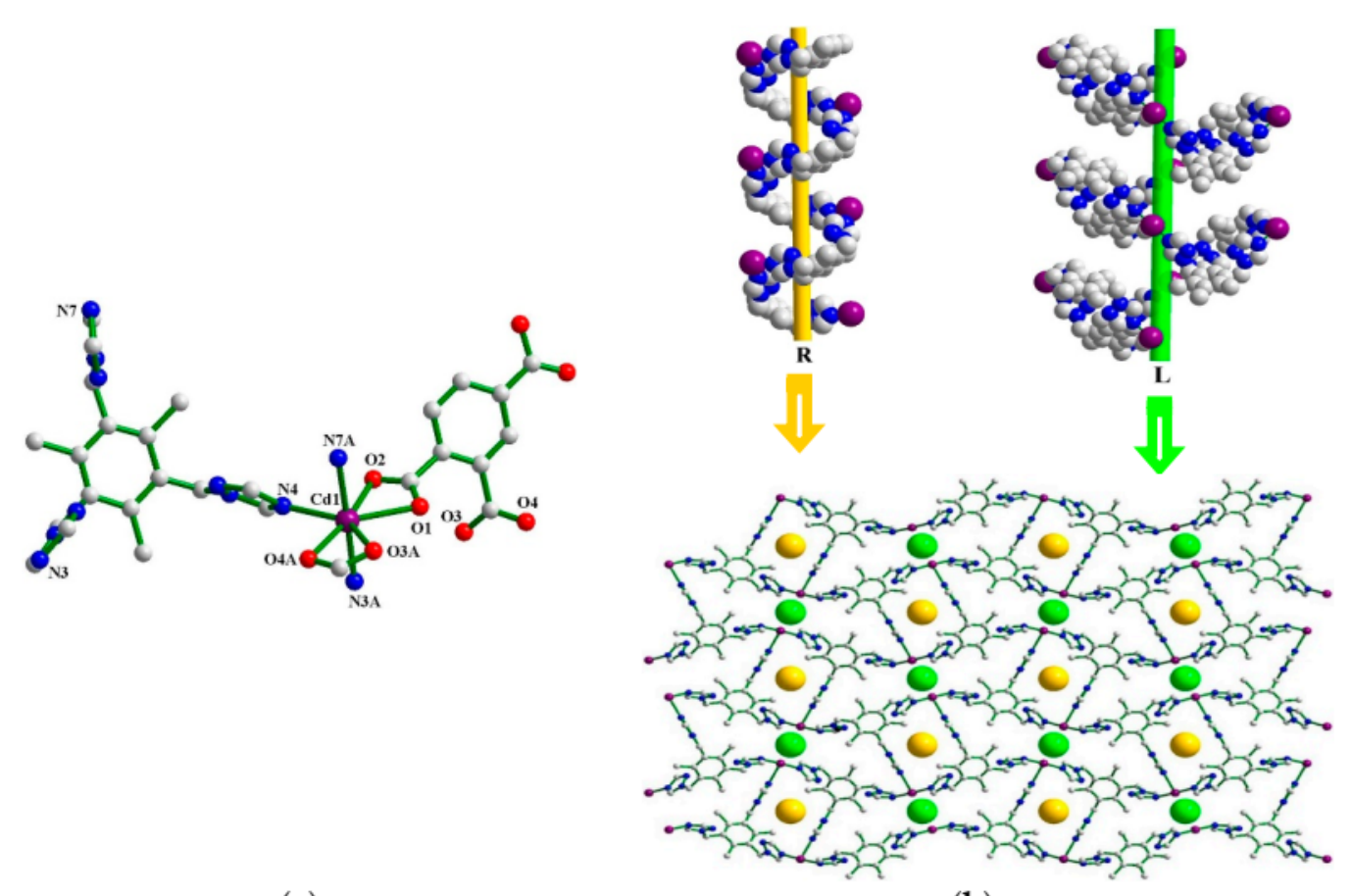

(a)

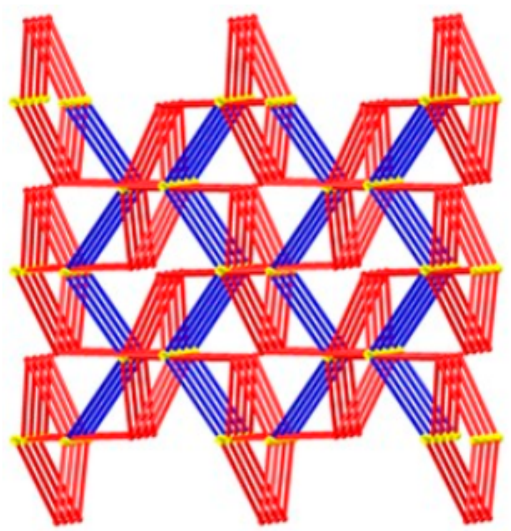

(b)

(c)

Figure 1. (a) Coordination environment of $\mathrm{Cd}(\mathrm{II})$ ion in 1. (b) The $3 \mathrm{D} \mathrm{Cd}(\mathrm{II}) / \mathrm{tttmb}$ architecture extended by alternately left- and right-handed helical chains. (c) Schematic representation of 1 with $\left(5^{3} \cdot 6 \cdot 7^{3} \cdot 8^{3}\right)\left(5^{2} \cdot 7\right)$ topology. Yellow node: Cd(II) ion; red stick: tttmb; blue stick: $\mathrm{Hbtc}^{2-}$.

The interesting feature of $\mathbf{1}$ is that the linkages between the Cd(II) ions and tttmb ligands form two kinds of helices. The tttmb ligands choose tridentate chelating coordination mode and bridge the adjacent $\mathrm{Cd}$ (II) ions to afford fascinating helical chains with left- and right-handed helical loops (Figure 1b). The pitches of left- and right-handed helix along the a axis both are equal to the length of the a axis. Each incompletely deprotonated $\mathrm{Hbtc}^{2-}$ anions displayed the bidentate coordinated mode coordinates of two $\mathrm{Cd}(\mathrm{II})$ centers. If the $\mathrm{Cd}$ (II) ion was taken as a 5-connected node, $\mathrm{tttmb}$ groups were regarded as 3-connected nodes and $\mathrm{Hbtc}^{2-}$ ligands were considered as linkers, $\mathbf{1}$ can be classified as a 3D structure with a point symbol of $\left(5^{3} \cdot 6 \cdot 7^{3} \cdot 8^{3}\right)\left(5^{2} \cdot 7\right)$ (Figure 1c).

Comparing 1 with $\left\{\left[\mathrm{Cd}_{2}(\mathrm{tttmb})_{2}(\mathrm{btc}) \mathrm{Cl}\right] \cdot 3 \mathrm{H}_{2} \mathrm{O}\right\}_{\mathrm{n}}(\mathbf{1 a})$ [21], both of which were prepared from similar starting reactants, some important differences were found as follows: (i) The conformation of tttmb ligand. The tttmb ligand adopts cis, cis, cis-conformation in $\mathbf{1}$, while cis, trans, trans-conformation in 1a. (ii) The coordination mode of $\mathrm{btc}^{3-}$ anions. It is bidentate in 1, but bidentate (carboxylate group at the 2,4-position) and monodentate (carboxylate group at the 1-position) in 1a. (iii) The coordination geometry of Cd(II) ions. 
It is in pentagonal bipyramid for $\mathbf{1}$, while in octahedron for 1a. (iv) The space group. Compound $\mathbf{1}$ crystallizes in the chiral orthorhombic space group, while $\mathbf{1 a}$ in the triclinic space group. (v) The synthesis conditions in $1, \mathrm{Cd}\left(\mathrm{CH}_{3} \mathrm{COO}\right)_{2} \cdot 2 \mathrm{H}_{2} \mathrm{O}$ was used as the metal source, while $\mathrm{CdCl}_{2} \cdot 2.5 \mathrm{H}_{2} \mathrm{O}$ used in $\mathbf{1 a}$. Pure $\mathrm{H}_{2} \mathrm{O}$ solvent was used for synthesis of $\mathbf{1}$, while the use of $\mathrm{NaOH}$ in $\mathrm{H}_{2} \mathrm{O}$ solvent for that of $\mathbf{1 a}$. Obviously, the addition of a strong base results in ligand $\mathrm{H}_{3}$ btc becoming completely deprotonated.

\subsubsection{Crystal Structure of 2}

Compound 2 crystallizes in the monoclinic space group C2/c and exhibits a twodimensional ladder-like layer structure. Each asymmetric unit of 2 contains one Cd(II) ion, one tttmb ligand, one m-phda ${ }^{2-}$ ligand, one coordinated water molecule, and two lattice water molecules. The $\mathrm{Cd}$ (II) ion exhibits a distorted pentagonal bipyramidal geometry with a $\mathrm{CdN}_{2} \mathrm{O}_{4}$ coordination sphere, completed by four oxygen atoms from two different $m$-phda ${ }^{2-}$ and one oxygen atom from a coordinated water molecule as well as two nitrogen atoms from two tttmb (Figure $2 \mathrm{a}$ ). The $\mathrm{Cd}-\mathrm{N}$ bonds lengths are 2.288 and $2.318 \AA$, while $\mathrm{Cd}-\mathrm{N}$ bond lengths are in the range of 2.354-2.569 $\AA$. The completely deprotonated $m$ phda $^{2-}$ ligands connect Cd(II) ions to form a 1D infinite helical chain with the right-handed and left-handed helices arranged alternately along the $b$ axis with the pitch of 12.889(3) $\AA$ (Figure 2b), which is further interconnected through the tttmb ligands to generate a 2D wave-like structure with $(4,4)$ sheets (Figure 2c).

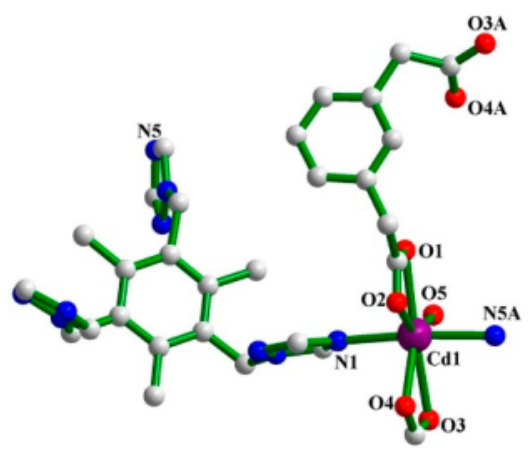

(a)

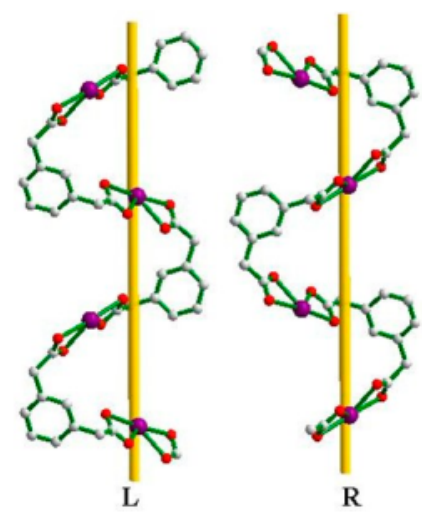

(b)

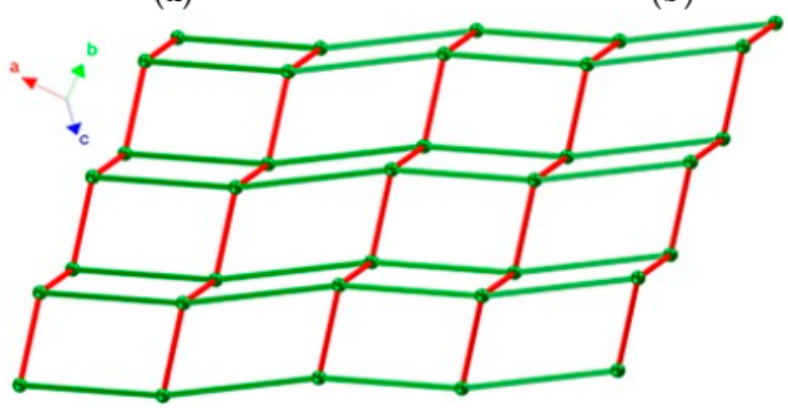

(c)

Figure 2. (a) Coordination environment of Cd(II) ions in 2 with hydrogen atoms omitted for clarity. (b) The right- and left-handed helical chains built by Cd(II) ions and $m$-phda ${ }^{2-}$ ligands. (c) View of the 2D ladder-like sheet with $4^{4}$ topology (green ball: $\mathrm{Cd}^{\mathrm{II}}$; Red line: $m$-phda ${ }^{2-}$; green line: $\mathrm{tttmb}$ ).

\subsubsection{Crystal Structure of 3}

Complex $\mathbf{3}$ also crystallizes in the monoclinic space group C2/c. As shown in Figure 3a, the $\mathrm{Cd}$ (II) ion is seven-coordinated, adopting a distorted pentagonal bipyramidal geometry via coordinating to four oxygen atoms from two $0-\mathrm{cpla}^{2-}$ anions and three nitrogen atoms from three tttmb ligands. The $\mathrm{Cd}-\mathrm{O}$ bond distances vary from 2.306 to $2.596 \AA$, while 
the $\mathrm{Cd}-\mathrm{N}$ bond lengths vary from 2.304 to $2.376 \AA$. In 3, tttmb adopts a cis, trans, transconformation. $\mathrm{N} 1$ and $\mathrm{N} 9$ atoms of $\mathrm{tttmb}$ connect $\mathrm{Cd}$ (II) ions to form a 1D wave-like chain. The 1D chain is further linked by N6 atoms of tttmb to expand to a 3D framework (Figure S1). The carboxylic acid groups of $o$-cpla ${ }^{2-}$ anions choose bidentate chelating coordination mode and bridge the adjacent $\mathrm{Cd}(\mathrm{II})$ ions to afford fascinating helical chains with left- and right-handed helical loops. (Figure $3 b$ ). The pitches of left- and right-handed helix along the $\mathrm{b}$ axis both are equal to the length of the $\mathrm{b}$ axis. In addition, the $\mathrm{Cd}(\mathrm{II}) / \mathrm{o-}^{-}$ cpla $^{2-}$ and Cd(II)-tttmb share Cd(II) ions to form a 3D architecture. Topological methods can provide better insight into such an elegant framework. The $\mathrm{Cd}$ (II) ion and tttmb ligand can be considered as five- and three-connected nodes, respectively, thus, the framework of 3 can be described as a rare $(2,3,5)$-connected net with the Schläfli symbol of $\left(6^{3}\right)\left(6^{6} \cdot 7 \cdot 8^{2} \cdot 10\right)$ (Figure 3c).

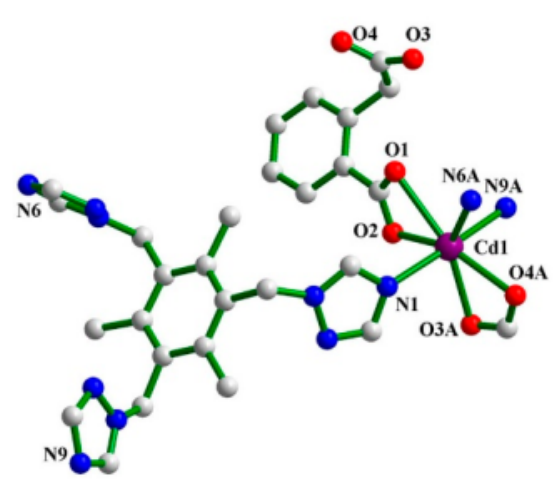

(a)

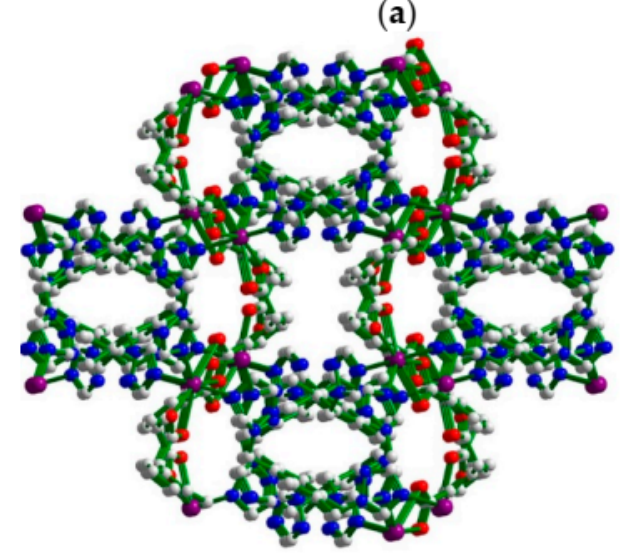

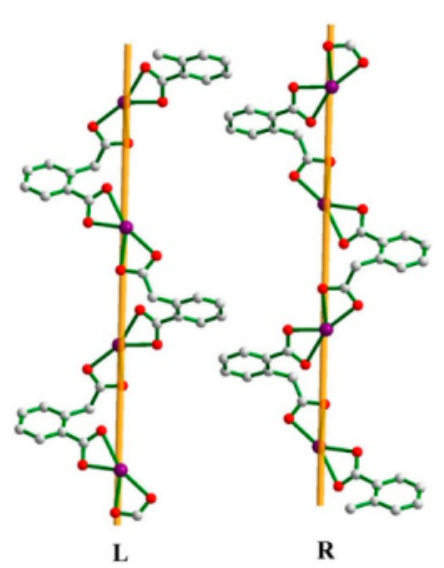

(b)

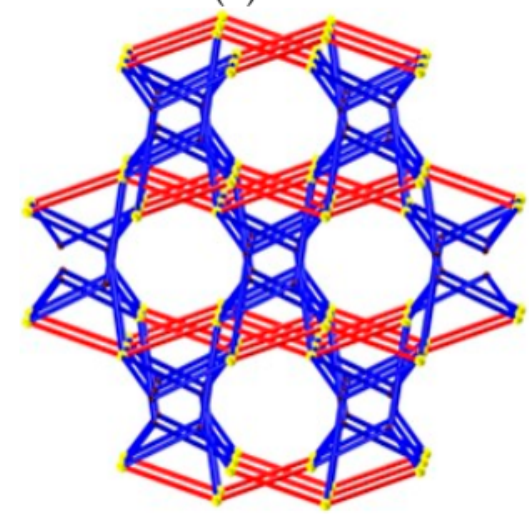

(c)

Figure 3. (a) Coordination environment of Cd(II) ions in $\mathbf{3}$ with hydrogen atoms omitted for clarity. (b) The Cd(II) /o-cpla ${ }^{2-}$ left- and right-handed helical chains along the $b$-axis. (c) Three-dimensional network and schematic representation of 3 . Blue line: tttmb; yellow node: $\mathrm{Cd}(\mathrm{II})$ ion; red line: $o$-cpla ${ }^{2-}$ group.

\subsubsection{Coordination Modes of $t \mathrm{ttmb}$ Ligand in 1-3}

According to the above structure description, the flexible tripod ligand tttmb has three different coordination modes in complexes 1-3, as depicted below in Figure 4. In 1, tttmb ligands adopt the $c i s, c i s, c i s-\mu_{3}$ coordination modes and coordinate with three $\mathrm{Cd}(\mathrm{II})$ atoms to form left- and right-handed helices (mode I in Figure 4). In 2, tttmb ligands show the cis, trans, trans- $\mu_{2}$ coordination modes (mode II in Figure 4 ) and coordinate with only two Cd(II) atoms to form 1D chains. In 3, tttmb ligands show similar cis, trans, trans-conformations, but act as a $\mu_{3}$-bridge linking three $\mathrm{Cd}$ (II) atoms to expand to a 3D framework (mode III in 
Figure 4). The results show that the coordination modes and the conformations of tttmb ligands obviously influence the structures of the complexes.
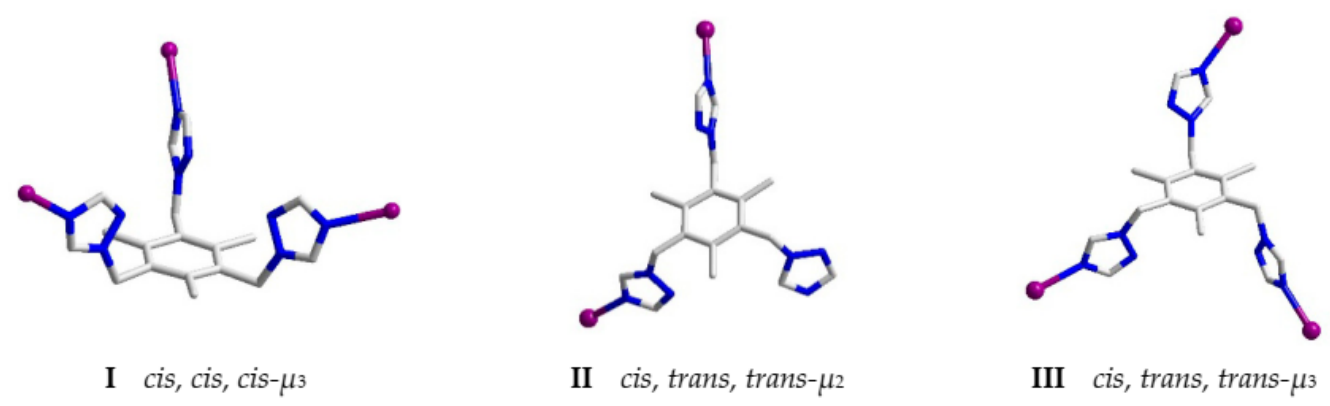

Figure 4. The different conformations of ligand tttmb in complexes.

\subsection{Powder X-ray Diffraction (PXRD) and Thermal Analyses}

The simulated and experimental PXRD patterns of compounds 1-3 (obtained at room temperature) are shown in Figure S2. Their peak positions correspond well with each other, indicating the phase purity of the solids.

The thermal stability of the complexes was investigated, as shown in Figure 5. For 1, there is an initial weight loss at $30.0-302.5{ }^{\circ} \mathrm{C}$, which arises from the loss of five water molecules (observed, 11.7\%; calculated, 11.6\%). Then the framework begins to decompose. For 2, weight loss of $8.0 \%$ occurs from 30.0 to $267.5^{\circ} \mathrm{C}$, which corresponds to the loss of one coordinated water molecule and two guest molecules (calculated, $7.8 \%$ ). Then the network begins to decompose. For 3, weight loss of $3.1 \%$ occurs from 30.0 to $145.0{ }^{\circ} \mathrm{C}$, which corresponds to the loss of solvent water molecules (calculated, 2.5\%).

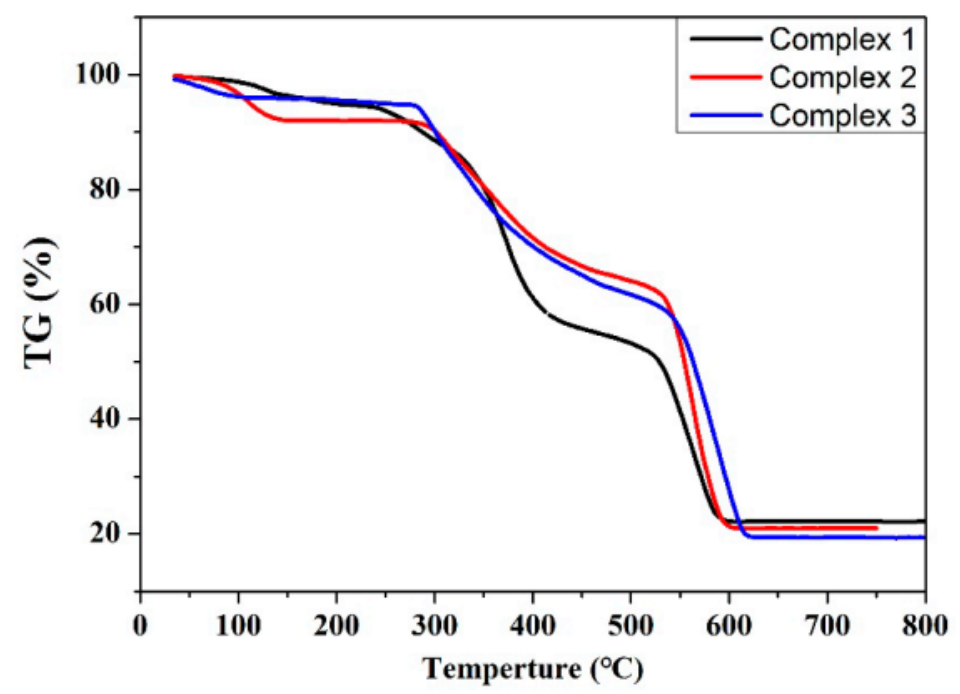

Figure 5. TG plots of complexes 1-3.

\subsection{Photoluminescence Properties}

The solid state photoluminescent behaviors of $\mathrm{Cd}(\mathrm{II})$ complexes 1-3, the free tttmb ligand and aromatic polycarboxylic acids $\left(\mathrm{H}_{3}\right.$ btc, $m-\mathrm{H}_{2}$ phda and $\left.o-\mathrm{H}_{2} \mathrm{cpla}\right)$ were investigated at ambient temperature under the same experimental conditions. The emission spectra of tttmb, $\mathrm{H}_{3}$ btc, $o-\mathrm{H}_{2}$ cpla and $m-\mathrm{H}_{2}$ phda are shown in Figure 6a. Intense bands were observed at $307 \mathrm{~nm}\left(\lambda_{\mathrm{ex}}=290 \mathrm{~nm}\right)$ for tttmb, $345 \mathrm{~nm}\left(\lambda_{\mathrm{ex}}=306 \mathrm{~nm}\right)$ for $\mathrm{H}_{3} \mathrm{btc}, 318 \mathrm{~nm}\left(\lambda_{\mathrm{ex}}=274 \mathrm{~nm}\right)$ for $o-\mathrm{H}_{2}$ cpla, and $358 \mathrm{~nm}\left(\lambda_{\mathrm{ex}}=314 \mathrm{~nm}\right)$ for $m-\mathrm{H}_{2}$ phda. The emission spectra of complexes 1-3 are shown in Figure $6 \mathrm{~b}$. Intense bands were observed at $454 \mathrm{~nm}\left(\lambda_{\mathrm{ex}}=348 \mathrm{~nm}\right)$ for $1,441 \mathrm{~nm}\left(\lambda_{\mathrm{ex}}=379 \mathrm{~nm}\right)$ for 2 , and $420 \mathrm{~nm}\left(\lambda_{\mathrm{ex}}=306 \mathrm{~nm}\right)$ for 3 . All these bands can be assigned to intraligand $\left(\pi^{*} \rightarrow \pi\right.$ or $\left.\pi^{*} \rightarrow \mathrm{n}\right)$ emission $[29,30]$. The emission bands of 3 
present red shifts $(62 \mathrm{~nm})$ in contrast to those of the free carboxylate ligands $\left(m-\mathrm{H}_{2}\right.$ phda). In view of similar excitation wavelengths for the complex and carboxylate ligands, the coordination of ligands with the $\mathrm{Cd}(\mathrm{II})$ ions can improve the conjugation degree of ligands, which may cause the red-shifts [31]. The different photoluminescent behaviors of $\mathbf{1}-\mathbf{3}$ with the same metal center and $\mathrm{N}$-donor ligand may be caused by the coordination environment, auxiliary aromatic polycarboxylate ligand.

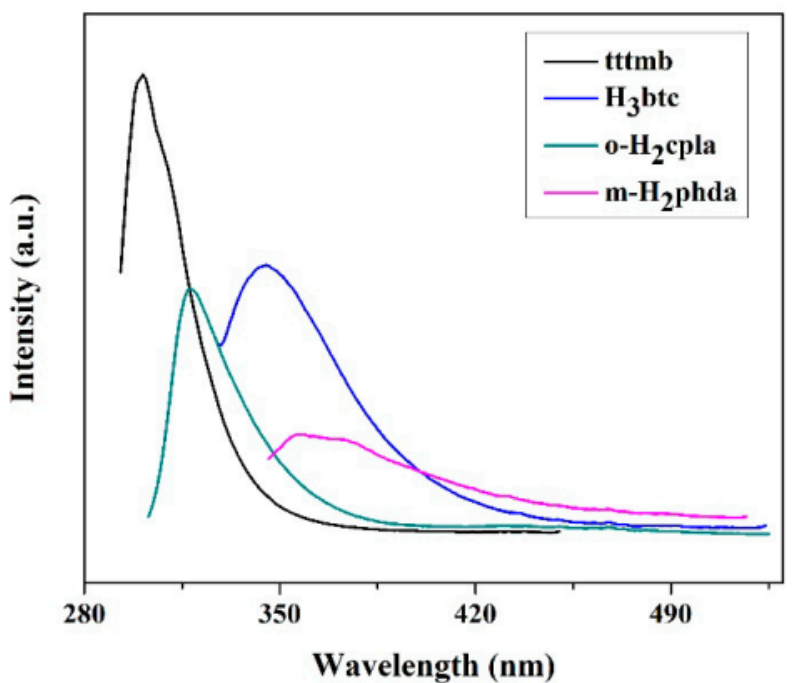

(a)

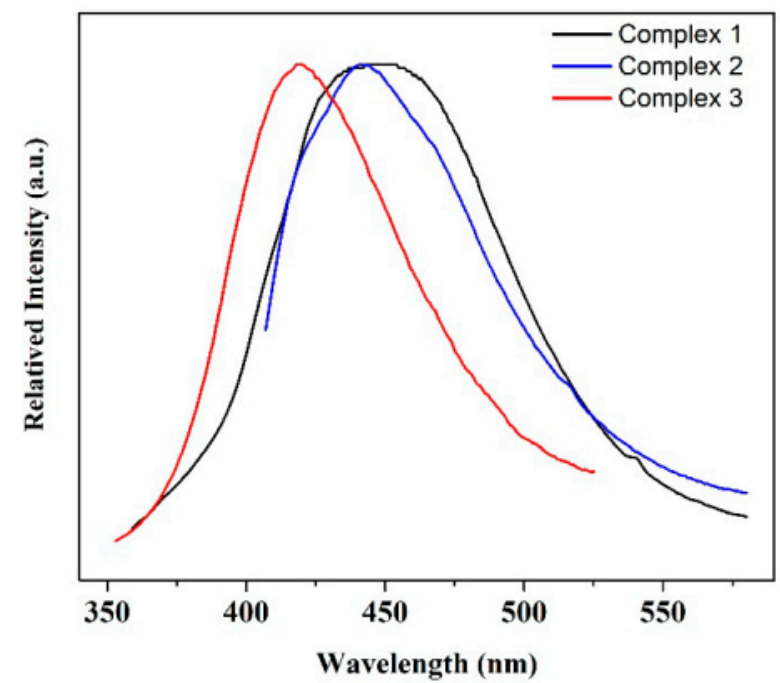

(b)

Figure 6. Solid-state photoluminescent spectra of (a) free ligands and (b) Complexs 1-3.

\section{Conclusions}

In summary, we have successfully synthesized three new $\mathrm{Cd}(\mathrm{II})$ complexes based on tttmb and different aromatic polycarboxylate ligands under hydro(solvo)thermal conditions. Our research demonstrates that tttmb is a good candidate for the assembling of coordination complexes with charming frameworks and topologies and has the potential to form chiral coordination complexes. Moreover, the structural versatility of $\mathbf{1}-\mathbf{3}$ shows that the coordination modes and the conformations of tttmb ligands can effectively tune the final structural features, as well as the aromatic polycarboxylate coligands. Subsequent studies about this flexible tripodal ligand $t t t m b$ are underway.

Supplementary Materials: The following supporting information can be downloaded at https: / /www.mdpi.com/article/10.3390/cryst12010053/s1. The description of the synthesis of 1,3,5tris(1,2,4-triazol-1-ylmethyl)-2,4,6-trimethylbenzene ligand. Table S1: Selected bond lengths ( $\AA$ ) and bond angles $\left(^{\circ}\right)$ for 1-3, Figure S1: The 3D framework of complex 3; Figure S2: Experimental (red) and simulated (black) PXRD patterns of compound 1-3.

Author Contributions: H.W. synthesized the three compounds. J.Q. performed the X-ray structure determination and analyzed the results. H.W. wrote the paper. All authors have read and agreed to the published version of the manuscript.

Funding: This research received no external funding.

Data Availability Statement: Crystallographic crystal data can be obtained free of charge via http:/ / www.ccdc.cam.ac.uk/conts/retrieving.html (accessed on 15 November 2021), or from the Cambridge Crystallographic Data Centre, 12 Union Road, Cambridge CB2 1EZ, UK; Fax: +44-1223-336-033; or e-mail: deposit@ccdc.cam.ac.uk.

Acknowledgments: This work was supported by key scientific research projects of higher education of Henan Province (16A150016) and Henan Province Natural Science Foundation (182300410237).

Conflicts of Interest: The authors declare no conflict of interest. 


\section{References}

1. Lin, Z.-J.; Lü, J.; Hong, M.; Cao, R. Metal-organic frameworks based on flexible ligands (FL-MOFs): Structures and applications. Chem. Soc. Rev. 2014, 43, 5867-5895. [CrossRef] [PubMed]

2. Ma, Y.-J.; Han, S.-D.; Mu, Y.; Pan, J.; Li, J.-H.; Wang, G.-M. Two Cobalt-Diphosphonates Templated by Long-Chain Flexible Amines: Synthesis, Structures, Proton Conductivity, and Magnetic Properties. Cryst. Growth Des. 2018, 18, 3477-3483. [CrossRef]

3. Hawes, C.S.; Hamilton, S.E.; Hicks, J.; Knowles, G.P.; Chaffee, A.L.; Turner, D.R.; Batten, S.R. Coordination Chemistry and Structural Dynamics of a Long and Flexible Piperazine-Derived Ligand. Inorg. Chem. 2016, 55, 6692-6702. [CrossRef] [PubMed]

4. Hawes, C.S.; Byrne, K.; Schmitt, W.; Gunnlaugsson, T. Flexible porous coordination polymers from divergent photoluminescent 4-oxo-1,8-naphthalimide ligands. Inorg. Chem. 2016, 55, 11570-11582. [CrossRef] [PubMed]

5. Qin, J.-H.; Qin, W.-J.; Xiao, Z.; Yang, J.-K.; Wang, H.-R.; Yang, X.-G.; Li, D.-S.; Ma, L.-F. Efficient energy-transfer-induced high photoelectric conversion in a dye-encapsulated ionic pyrene-based metal-organic framework. Inorg. Chem. 2021, 60, 18593-18597. [CrossRef]

6. Kang, Y.S.; Lu, Y.; Chen, K.; Zhao, Y.; Wang, P.; Sun, W.Y. Metal-organic frameworks with catalytic centers: From synthesis to catalytic application. Coord. Chem. Rev. 2019, 378, 262-280. [CrossRef]

7. Zhai, Q.G.; Bu, X.; Mao, C.; Zhao, X.; Feng, P. Systematic and dramatic tuning on gas sorption performance in heterometallic metal-organic frameworks. J. Am. Chem. Soc. 2016, 138, 2524-2527. [CrossRef]

8. Qin, J.-H.; Huang, Y.-D.; Zhao, Y.; Yang, X.-G.; Li, F.-F.; Wang, C.; Ma, L.-F. Highly Dense Packing of Chromophoric Linkers Achievable in a Pyrene-Based Metal-Organic Framework for Photoelectric Response. Inorg. Chem. 2019, 58, 15013-15016. [CrossRef]

9. Yang, X.-G.; Ma, L.-F.; Yan, D.-P. Facile synthesis of 1D organic-inorganic perovskite micro-belts with high water stability for sensing and photonic applications. Chem. Sci. 2019, 10, 4567-4572. [CrossRef]

10. Qin, J.-H.; Xu, P.; Huang, Y.-D.; Xiao, L.-Y.; Lu, W.; Yang, X.-G.; Ma, L.; Zang, S.-Q. High loading of Mn(II)-metalated porphyrin in MOF for photocatalytic $\mathrm{CO}_{2}$ reduction in gas-solid condition. Chem. Commun. 2021, 57, 8468-8471. [CrossRef]

11. Bai, S.-Q.; Young, D.J.; Hor, T.S.A. Nitrogen-rich azoles as ligand spacers in coordination polymers. Chem.-Asian J. 2011, 6, 292-304. [CrossRef]

12. Zhao, S.; Wu, J.; Chen, W. Organometallic chemistry of bis(N-heterocyclic carbene) ligands containing a heteroarene spacer. $J$. Organomet. Chem. 2017, 848, 249-280. [CrossRef]

13. Liu, X.; Du, L.; Li, R.; Ma, N.; You, M.; Feng, X. Different effects in the selective detection of aniline and Fe ${ }^{3+}$ by lanthanide-based coordination polymers containing multiple reactive sites. CrystEngComm 2020, 22, 2837-2844. [CrossRef]

14. Qin, J.-H.; Zhang, H.; Sun, P.; Huang, Y.-D.; Shen, Q.; Yang, X.-G.; Ma, L.-F. Ionic liquid induced highly dense assembly of porphyrin in MOF nanosheets for photodynamic therapy. Dalton Trans. 2020, 49, 17772-17778. [CrossRef]

15. Liu, X.; Zhang, X.; Li, R.; Du, L.; Feng, X.; Ding, Y. A highly Sensitive and Selective "Turn off-on" Fluorescent Sensor Based on Sm-MOF for the Detection of Tertiary Butylhydroquinone. Dyes Pigm. 2020, 178, 108347. [CrossRef]

16. Xue, X.-F.; Liu, Y.-Q.; Liu, Q.; Wang, X.-Y.; Li, W. Four Novel Coordination Polymers Based on Flexible 1,4-bis(1,2,4-triazol1-ylmethyl)benzene Ligand: Synthesis, Structure, Luminescence and Magnetic Properties. J. Clust. Sci. 2019, 30, 777-787. [CrossRef]

17. Liu, K.; Shi, W.; Cheng, P. The coordination chemistry of $\mathrm{Zn}(\mathrm{II}), \mathrm{Cd}(\mathrm{II})$ and $\mathrm{Hg}(\mathrm{II})$ complexes with 1,2,4-triazole derivatives. Dalton Trans. 2011, 40, 8475-8490. [CrossRef]

18. Yang, P.; Wu, X.X.; Huo, J.Z.; Ding, B.; Wang, Y.; Wang, X.G. Hydrothermal synthesis and characterization of a series of luminescent $\mathrm{Zn}(\mathrm{II})$ and $\mathrm{Cd}(\mathrm{II})$ coordination polymers with the new versatile multidentate ligand 1,3-di-(1,2,4-triazol-4yl)benzene. CrystEngComm 2013, 15, 8097-8109. [CrossRef]

19. Gautier, R.; Clérac, R. Tuning the Crystal Structure Dimensionality of Cobalt(II)/1,2,4-Triazole Complexes. Cryst. Growth Des. 2017, 17, 864-869. [CrossRef]

20. Zhang, M.N.; Fan, T.T.; Wang, Q.S.; Han, H.L.; Li, X. Zn/Cd/Cu-frameworks constructed by 3,3'-diphenyldicarboxylate and 1,4-bis(1,2,4-triazol-1-yl)butane: Syntheses, structure, luminescence and luminescence sensing for metal ion in aqueous medium. J. Solid State Chem. 2018, 258, 744-752. [CrossRef]

21. Wang, X.; Liu, Y.; Xu, C.; Guo, Q.; Hou, H.; Fan, Y. Series of Cd(II) Metal-Organic Frameworks Based on a Flexible Tripodal Ligand and Polycarboxylate Acids: Syntheses, Structures, and Photoluminescent Properties. Cryst. Growth Des. 2012, 12, 2435-2444. [CrossRef]

22. Wu, Q.; Han, Y.B.; Shao, Z.C.; Li, J.X.; Hou, H.W. Stable Fe(II)-based coordination polymers: Synthesis, structural diversity and catalytic applications in homo-coupling reactions. Dalton Trans. 2018, 47, 8063-8069. [CrossRef] [PubMed]

23. Zhang, Z.-Y.; Zhang, G.-D.; Sheng, X.-X.; Ding, Q.-W.; Bai, Y.-Z.; Su, Y.; Liu, H.-K.; Su, Z. Efficient MO dye degradation catalyst of $\mathrm{Cu}(\mathrm{I})$-based coordination complex from dissolution-recrystallization structural transformation. Cryst. Growth Des. 2021, 21, 333-343. [CrossRef]

24. Chang, X.-H.; Zhao, Y.; Han, M.-L.; Ma, L.-F.; Wang, L.-Y. Five Cd(II) coordination polymers based on 2,3' ,5,5'-biphenyltetracarboxylic acid and N-donor coligands: Syntheses, structures and fluorescent properties. CrystEngComm 2014, 16, 6417-6424. [CrossRef]

25. Zhang, F.; Hou, L.; Zhang, W.Y.; Yan, Y.T.; Wu, Y.L.; Yang, R.F.; Cao, F.; Wang, Y.Y. Two metal-organic frameworks based on a flexible benzimidazole carboxylic acid ligand: Selective gas sorption and luminescence. Dalton Trans. 2017, 46, 15118-15123. [CrossRef] 
26. Miao, S.-B.; Li, Z.-H.; Xu, C.-Y.; Ji, B.-M. A New 3-Fold Interpenetrating 3D Zn(II) Metal-organic Framework: Synthesis, Structure and Luminescent Property. Chin. J. Struct. Chem. 2016, 35, 1960-1966.

27. Dolomanov, O.V.; Bourhis, L.J.; Gildea, R.J.; Howard, J.A.K.; Puschmann, H. OLEX2: A complete structure solution, refinement and analysis program. J. Appl. Cryst. 2009, 42, 342-346. [CrossRef]

28. Sheldrick, G.M. Crystal structure refinement with SHELXL. Acta Cryst. 2015, 71, 3-8.

29. Meng, W.; Liu, Y.; Zhao, Y.; Hou, H.; Fan, Y. Coordination polymers based on a flexible tripodal ligand and aromatic dicarboxylate anions: Syntheses, structures and fluorescent properties. Polyhedron 2013, 52, 1219-1226. [CrossRef]

30. Wang, K.; Tang, H.; Zhang, D.; Ma, Y.; Wang, Y. Selective and recyclable sensing of aqueous phase 2,4,6-Trinitrophenol (TNP) based on Cd(II) coordination polymer with Zwitterionic ligand. Crystals 2018, 8, 456. [CrossRef]

31. Miao, S.-B.; Sun, X.-J.; Wang, K.-X.; Xu, C.-Y.; Li, Z.-H.; Wang, Z.-Q. Effect of Charge on the Structures of Zn(II) Coordination Polymers with Triazole-carboxylate Ligands: Syntheses, Structures, and Luminescent Properties. Crystals 2018, 8, 312. [CrossRef] 